\title{
Correlation between the dynamics of polar nanoregions and temperature evolution of central peaks in $\mathrm{Pb}\left[\left(\mathrm{Zn}_{1 / 3} \mathrm{Nb}_{2 / 3}\right)_{0.91} \mathrm{Ti}_{0.09}\right] \mathrm{O}_{3}$ ferroelectric relaxors
}

\author{
Jae-Hyeon $\mathrm{Ko}^{\mathrm{a})}$ \\ Department of Physics, Hallym University, 39 Hallymdaehakgil, Chuncheon, Gangwondo 200-702, Korea \\ Do Han Kim ${ }^{\text {b) }}$ and Seiji Kojima ${ }^{\mathrm{c})}$ \\ Institute of Materials Science, University of Tsukuba, Tsukuba city, Ibaraki 305-8573, Japan
}

(Received 10 January 2007; accepted 6 February 2007; published online 13 March 2007)

\begin{abstract}
Acoustic properties of $\mathrm{Pb}\left[\left(\mathrm{Zn}_{1 / 3} \mathrm{Nb}_{2 / 3}\right)_{0.91} \mathrm{Ti}_{0.09}\right] \mathrm{O}_{3}$ have been investigated in a wide temperature range. The sound velocity of acoustic phonons showed a deviation from its high-temperature linear behavior at the Burns temperature $T_{B} \sim 730 \mathrm{~K}$. Upon cooling, acoustic properties exhibited significant changes at about $550 \mathrm{~K}$ in polarization state and width of the central peak in addition to changes in frequency and damping of acoustic phonons. This finding suggests that, besides $T_{B}$, another intermediate characteristic temperature exists in the evolution of relaxor dynamics, which might be related to the formation of long-lived polar nanoregions and associated local strain fields. (C) 2007 American Institute of Physics. [DOI: 10.1063/1.2713170]
\end{abstract}

Lead-based complex perovskite relaxor ferroelectrics have attracted great attention from the viewpoint of fundamental physics due to their complex structures and dynamic behaviors. $\mathrm{Pb}\left(\mathrm{Mg}_{1 / 3} \mathrm{Nb}_{2 / 3}\right) \mathrm{O}_{3}$, for example, shows a diffuse, frequency-dependent broad dielectric maximum, broad distribution of relaxation times with divergent leading edge on cooling, no macroscopic symmetry change with the existence of nanosized polar regions (PNRs) below a certain temperature, the so-called Burns temperature $\left(T_{B}\right){ }^{1,2}$ The temperature evolution of the relaxor dynamics has been thought to be correlated with the formation of PNRs and growing interactions between them upon cooling. In spite of all the experimental and theoretical efforts on relaxors, including random-field model, ${ }^{3}$ dipolar glass mode, ${ }^{4}$ and recent spherical random bond-random field model, ${ }^{5}$ the microscopic origin of their complex behaviors is still not clear.

Recent systematic Raman studies on $\mathrm{Pb}\left(\mathrm{Zn}_{1 / 3} \mathrm{Nb}_{2 / 3}\right) \mathrm{O}_{3}$ (PZN) and $\mathrm{Pb}\left[\left(\mathrm{Zn}_{1 / 3} \mathrm{Nb}_{2 / 3}\right)_{1-x} \mathrm{Ti}_{x}\right] \mathrm{O}_{3}(\mathrm{PZN}-x \mathrm{PT})$ with $x$ $=4.5 \%$ suggested another characteristic temperature, denoted as $T_{d}$, which is located far below $T_{B}{ }^{6,7}$ This intermediate temperature is characterized by anomalies in the temperature dependences of frequency and/or intensity of optic modes and central peaks (CPs) observed in Raman spectra. It has been suggested as the onset temperature of long-lived correlations between off-center ions resulting in the formation of permanent PNRs. ${ }^{6}$ This result is consistent with that obtained from acoustic emission measurement, which showed clear, strong signals near $510 \mathrm{~K}$ in PZN and PZN-9\%PT, in addition to the acoustic emission activity near $T_{B} \sim 730 \mathrm{~K}$ in both components. ${ }^{8}$

Local phase transformation near $T_{d}$ suggested above should be accompanied by local strain fields, which would bridge the long-lived correlations of off-center ions and the formation of permanent PNRs in PZN- $x \% \mathrm{PT}^{6}$ In this re-

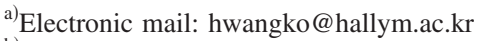

${ }^{b)}$ Present address: PDP Research Laboratory, PDP Division, LG Electronics Inc. 184, Gongdan-dong, Gumi, GyoungBuk 730-702, Korea.

${ }^{c)}$ Electronic mail: kojima@bk.tsukuba.ac.jp
}

spect, inelastic Brillouin light scattering can be a very useful tool because it can directly probe acoustic modes which can be coupled to the PNRs through electristrictive coupling, and characteristic time scale of CPs is located in the Brillouin frequency window. Previous Brillouin scattering studies on PZN- $x \%$ PT have mainly been focused on the diffuse phase transitions near the dielectric maxima and thus been carried out in limited temperature ranges much lower than $T_{B}{ }^{9-15}$ The present letter reports systematic investigation of the temperature dependence of acoustic modes and CPs of PZN9\% PT in a wide temperature range between 300 and $900 \mathrm{~K}$ in order to find out whether $T_{d}$ has a real physical meaning in the temperature evolution of relaxor dynamics.

PZN-9\%PT single crystals were grown by the Bridgman method at Shanghai Institute of Ceramics and cut for obtaining pseudocubic crystal orientations. The Brillouin scattering system with a $3+3$ pass Sandercock-type tandem FabryPérot interferometer has been used to measure the Brillouin spectra. Both the polarized (VV) and depolarized (VH) spectra were obtained during the cooling process. A special rightangle scattering geometry was used for the measurement without any significant disturbance of the elastic scattering. ${ }^{11}$ In this scattering geometry, the phonon propagation direction is along the $[100]_{c}$.

Figures 1(a) shows the Brillouin spectra measured in a narrow frequency windows of $\pm 20 \mathrm{GHz}$ at $453 \mathrm{~K}$. As can be seen, the transverse acoustic (TA) mode appears in the VH spectrum while the longitudinal acoustic (LA) mode is shown in the VV spectrum. The sound velocities of the TA and LA modes correspond to the elastic stiffness coefficients $c_{44}$ and $c_{11}$, respectively, in the cubic coordinates. The CP was investigated in a much wider frequency range of $\pm 450 \mathrm{GHz}$, which is shown in Fig. 1(b) in a semilog plot.

The temperature dependences of the Brillouin shift $\left(\nu_{B}\right)$ and the full width at half maximum (FWHM) of the LA and TA phonons have been obtained and plotted in Figs. 2(a) and 2(b), respectively. $\nu_{B}$ 's of both modes show an almost linear behavior in the high-temperature range above $T_{B}$. Since no coupling exists between the acoustic modes and other de- 


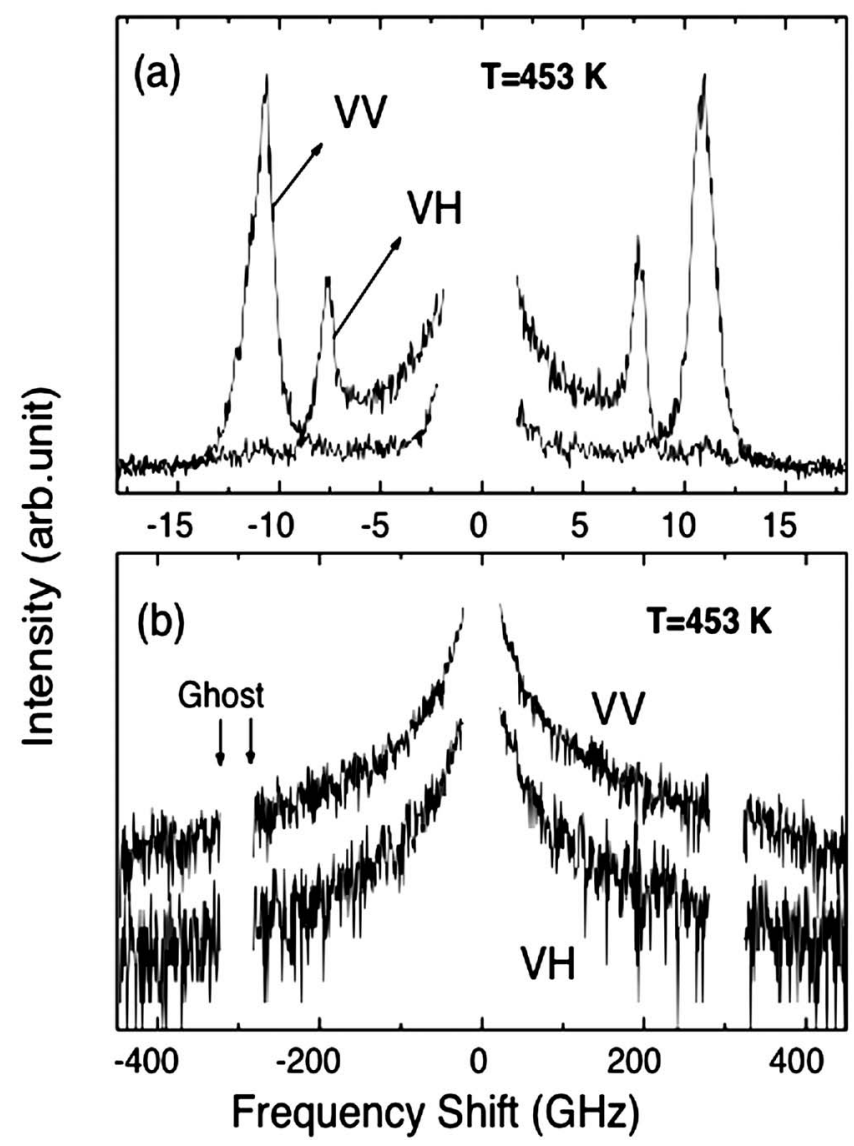

FIG. 1. Polarized (VV) and depolarized (VH) Brillouin spectra measured in (a) narrow and (b) wide frequency ranges at $453 \mathrm{~K}$.

grees of freedom related to any phase transformation in this high-temperature range, the temperature dependence of the phonon frequency will be mainly dominated by the lattice anharmonicity. ${ }^{16} \nu_{B}$ of LA mode deviates from its hightemperature linearity near $720 \mathrm{~K}\left(\sim T_{B}\right)$ at which the FWHM shows a change in slope. The correlation between $T_{B}$ and the anomaly in $\nu_{B}$ of LA phonon has also been observed in $\mathrm{Pb}\left(\mathrm{Mg}_{1 / 3} \mathrm{Ta}_{2 / 3}\right) \mathrm{O}_{3}$ and lanthanum lead zirconate titanate relaxors. ${ }^{17,18}$ After reaching a maximum, significant softening in $\nu_{B}$ begins to occur at $\sim 570$ and $\sim 510 \mathrm{~K}$ for LA and TA modes, respectively. The softening of $\nu_{B}$ of both modes is accompanied by the increase of acoustic damping represented by the phonon linewidth. Both modes experience drastic changes in $\nu_{B}$ and FWHM at the cubic-to-tetragonal phase transition temperature $\left(T_{C-T}\right)$ near $430 \mathrm{~K}$.

$\mathrm{CP}$ was fitted by a single Lorentzian centered at zero. Figures 3(a) and 3(b) show temperature dependences of the integrated intensity and the FWHM of CPs $\left(\Delta_{\mathrm{CP}}\right)$, respectively, measured at both polarized and depolarized scattering geometries. Polarized component of the CP persists at high temperatures even above $T_{B}$. In contrast, there is almost no depolarized component of the $\mathrm{CP}$ in this high-temperature range above $600 \mathrm{~K}$. Upon further cooling, CP exhibits drastic changes. First, the half-width of VV component decreases from $\sim 700 \mathrm{GHz}$ at $595 \mathrm{~K}$ down to $\sim 70 \mathrm{GHz}$ near $T_{C-T}$ while its intensity increases. Second, the VH component begins to appear near $550 \mathrm{~K}$, denoted as $T_{d}$ henceforth, pointing out that off-diagonal components of the polarizability tensor develop around this temperature. Below this temperature, the width of this depolarized CP decreases with lowerDownloaded 30 Nov 2009 to 130.158 .56 .186 . Redistribution subject

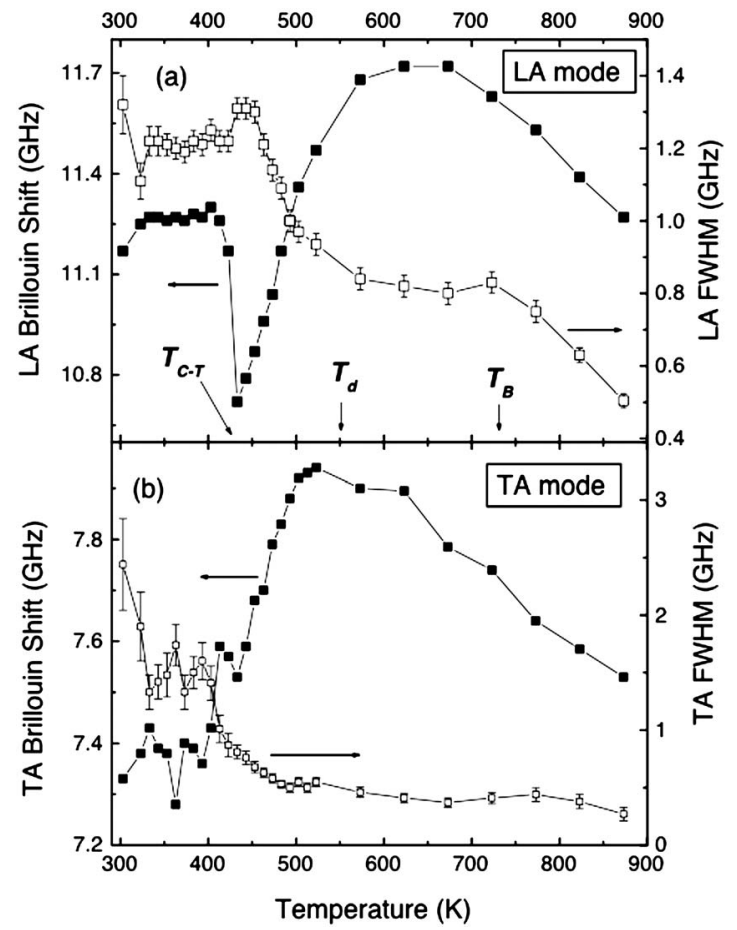

FIG. 2. Temperature dependences of the Brillouin shift $\left(\nu_{B}\right)$ and the full width at half maximum (FWHM) of the (a) LA and (b) TA phonons. Regarding the meaning of $T_{B}, T_{d}$, and $T_{C-R}$, see the text.

ing temperature and its intensity increases with decreasing temperature from $550 \mathrm{~K}$ and shows a sudden jump at $T_{C-T}$.

Although CPs can be related to several origins in condensed matters, CPs in ferroelectrics can arise from soft transverse-optic (TO) phonon, or from some relaxational en-

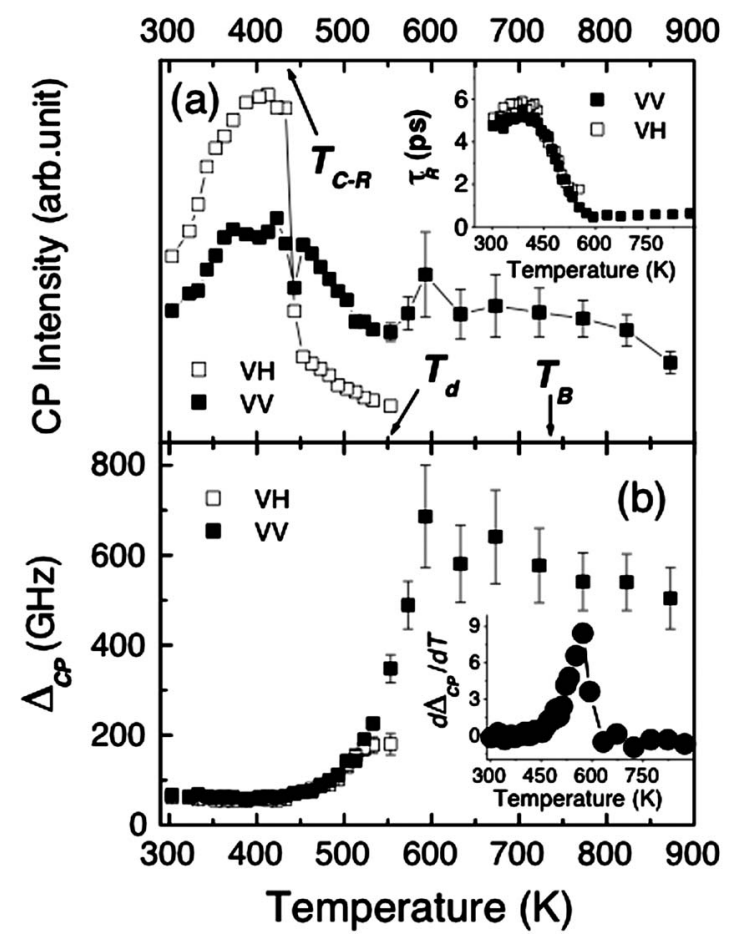

FIG. 3. Temperature dependences of (a) the integrated intensity and (b) the FWHM of CPs measured at polarized and depolarized scattering geometries. The inset of (a) shows the temperature dependence of relaxation times estimated from the width of polarized and depolarized CPs. The inset of (b) shows the rate of change in the half-width of the VV component of $\mathrm{CP}$ with respect to the temperature (the unit, $\mathrm{GHz} / \mathrm{K}$, was omitted for better clarity). respect to the temperature (the unit, GHz/K, was omitted for better clarity).
o AlP license or copyright; see http://apl.aip.org/apl/copyright.jsp 
tities, or from the coupling between them. Since previous studies reported that the TO phonons in PZN and PZN-8\%PT are overdamped below $T_{B},{ }^{19,20}$ the most probable origin of CPs in PZN-9\%PT might be the relaxational dynamics of PNRs. ${ }^{9-15}$ The CP dynamics is dominated by the reorientation of the PNRs, and the CP width can be used to estimate the approximate relaxation time of the PNRs. Temperature dependences of the acoustic modes and CP of PZN-9\%PT revealed three characteristic temperature regions above $T_{C-T}$ divided by two characteristic temperatures, $T_{B}$ and $T_{d}$.

Above $T_{B} \sim 730 \mathrm{~K}, c_{44}$ and $c_{11}$ are changing according to the usual lattice anharmonicity and only the polarized CP can be seen. In this high-temperature range where the off-center ions can reorient freely without restriction, rapid $180^{\circ}$ reorientations of off-center ions, probably smaller, lighter $\mathrm{Nb}$ ions, ${ }^{21}$ are expected to contribute to the $\mathrm{VV}$ component of the CP due to symmetry consideration. ${ }^{6}$ Near $T_{B}, \nu_{B}$ 's of both modes deviate from their high-temperature liner behaviors, but there is no change in the $\mathrm{VV}$ component of the $\mathrm{CP}$ and VH component cannot be seen. Between $T_{B}$ and $550 \mathrm{~K}, \nu_{B}$ 's exhibit broad maxima with small acoustic dampings, and the change in the intensity and $\Delta_{\mathrm{CP}}$ of the $\mathrm{VV}$ component of $\mathrm{CP}$ is negligible.

Upon further cooling, $\mathrm{VH}$ component of the $\mathrm{CP}$ appears at about $550 \mathrm{~K}$ and both components of $\mathrm{CP}$ become narrower significantly indicating marked slowing down of the dynamics of PNRs. This is consistent with the fact that all previous Brillouin studies on PZN- $x \%$ PT found that the VH component of CP appears at temperatures much lower than $T_{B} .{ }^{9-15}$ The temperature dependence of the relaxation times $\tau_{R}$ of PNRs estimated from the half-width of CP is plotted in the inset of Fig. 3(a). $\tau_{R}$ estimated from VV component is approximately 0.6 ps above $600 \mathrm{~K}$ and increases markedly below this temperature. The change of $\Delta_{\mathrm{CP}}$ of the VV component of $\mathrm{CP}$ with respect to the temperature, i.e., $d \Delta_{\mathrm{CP}} / d T$ becomes maximum at $\sim T_{d}$, as can be seen from the inset in Fig. 3(b). This means that the rate of change in $\tau_{R}$ becomes fastest near this temperature. In addition, $\tau_{R}$ estimated from the width of the VH component exhibits almost the same temperature dependence as that from the VV component below $550 \mathrm{~K}$, which indicates that the VV and VH components of CP are strongly coupled below $T_{d}$. The appearance of the $\mathrm{VH}$ component at a certain characteristic temperature indicates restriction of the free reorientation of off-center ions and thus local symmetry lowering in PNRs occur. ${ }^{22}$

The present study revealed a characteristic temperature $T_{d} \sim 550 \mathrm{~K}$ in the temperature evolution of acoustic properties of PZN-9\%PT. $T_{d}$ is characterized by (i) the appearance of the $\mathrm{VH}$ component of $\mathrm{CP}$, (ii) significant decrease in $\Delta_{\mathrm{CP}}$ of both VV and VH components of CP implying strong coupling between them and slowing down of the dynamics of PNRs, (iii) onset of significant softening in $c_{44}$ and $c_{11}$, and (iv) increase of the acoustic damping in both LA and TA modes. This indicates that the coupling between the dynamics of PNRs and acoustic modes becomes substantial at $T_{d}$, since the electrostrictive coupling is responsible for the softening of the sound velocities and the increase in acoustic damping. In addition, the increase of the intensity of $\mathrm{CP}$ and their narrowing in width signifies that the density of PNRs and interactions between them begin to grow at this temperature. Therefore, it may be indicated that $T_{d}$ is a characteristic temperature at which long-lived PNRs begin to form inducing local strain fields, which will be coupled to the acoustic modes, resulting in the changes of acoustic properties observed in this study.

To summarize, the temperature dependence of acoustic properties of $\mathrm{PZN}-9 \% \mathrm{PT}$ has been investigated in a wide temperature range by Brillouin light scattering. At a certain temperature of $T_{d} \sim 550 \mathrm{~K}$ located below $T_{B}$, depolarized $\mathrm{CP}$ began to appear, and the slowing down of PNRs represented by the narrowing $\mathrm{CP}$ concomitant with the drastic softening of $c_{44}$ and $c_{11}$ was observed upon cooling from this temperature. Increasing Brillouin linewidth of LA and TA phonons below $T_{d}$ reflects the fluctuation damping arising from the electrostrictive coupling of the strain to the square of the local polarization of PNRs. The present result suggests a clear experimental evidence of the existence of the intermediate characteristic temperature of $\sim 550 \mathrm{~K}$ in PZN-9\%PT at which significant changes in acoustic properties are brought about, and this temperature might indicate that long-lived PNRs and associated local strain fields are formed not at $T_{B} \sim 730 \mathrm{~K}$ but at a much lower temperature $T_{d} \sim 550 \mathrm{~K}$.

This work was supported by the Korea Research Foundation Grant funded by the Korean Government (MOEHRD, Basic Research Promotion Fund) (KRF-2006-003-C00088) and by MEXT, Japan, Grant-in-Aid for Scientific Research (A), 2006, No.16204032.

${ }^{1}$ L. E. Cross, Ferroelectrics 76, 241 (1987).

${ }^{2}$ G. Burns and F. H. Dacol, Ferroelectrics 104, 25 (1990).

${ }^{3}$ V. Westphal, W. Kleemann, and M. D. Glinchuk, Phys. Rev. Lett. 68, 847 (1992).

${ }^{4}$ D. Viehland, S. J. Jang, L. E. Cross, and M. Wuttig, J. Appl. Phys. 68, 2916 (1990).

${ }^{5}$ R. Pirc and R. Blinc, Phys. Rev. B 60, 13470 (1990).

${ }^{6}$ J. Toulouse, F. Jiang, O. Svitelskiy, W. Chen, and Z.-G. Ye, Phys. Rev. B 72, 184106 (2005).

${ }^{7}$ O. Svitelskiy, D. La-Orauttapong, J. Toulouse, W. Chen, and Z.-G. Ye, Phys. Rev. B 72, 172106 (2005).

${ }^{8}$ E. Dul'kin, M. Roth, P.-E. Janolin, and B. Dkhil, Phys. Rev. B 73, 012102 (2006).

${ }^{9}$ M. H. Kuok, S. C. Ng, H. J. Fan, M. Iwata, and Y. Ishibashi, Solid State Commun. 118, 169 (2001).

${ }^{10}$ M. H. Kuok, S. C. Ng, H. J. Fan, M. Iwata, and Y. Ishibashi, Appl. Phys. Lett. 78, 1727 (2001).

${ }^{11}$ Y. Gorouya, Y. Tsujimi, Y. Yamashita, T. Ifukube, and T. Yagi, Ferroelectrics 266, 165 (2002).

${ }^{12}$ Y. Gorouya, Y. Tsujimi, M. Iwata, and Y. Yagi, Appl. Phys. Lett. 83, 1358 (2003).

${ }^{13}$ D. H. Kim, J.-H. Ko, C. D. Feng, and S. Kojima, Appl. Phys. Lett. 87, 072908 (2005).

${ }^{14}$ D. H. Kim, J.-H. Ko, C. D. Feng, and S. Kojima, J. Appl. Phys. 98, 044106 (2005).

${ }^{15}$ Y. Nakata, Y. Tsujimi, K. Katsuraya, M. Iwata, and T. Yagi, Appl. Phys. Lett. 89, 022903 (2006).

${ }^{16}$ E. Courtens, R. Vacher, and Y. Dagorn, Phys. Rev. B 33, 7625 (1986).

${ }^{17}$ J.-H. Ko, S. Kojima, and S. G. Lushnikov, Appl. Phys. Lett. 82, 4128 (2003).

${ }^{18}$ G. Shabbir, J.-H. Ko, S. Kojima, and Q.-R. Yin, Appl. Phys. Lett. 82, 4696 (2003).

${ }^{19}$ P. M. Gehring, S.-E. Park, and G. Shirane, Phys. Rev. Lett. 84, 5216 (2000).

${ }^{20}$ P. M. Gehring, S.-E. Park, and G. Shirane, Phys. Rev. B 63, 224109 (2001).

${ }^{21}$ B. E. Vugmeister, Phys. Rev. B 73, 174117 (2006).

${ }^{22}$ O. Svitelskiy and J. Toulouse, J. Phys. Chem. Solids 64, 665 (2003). 